Table 1

\begin{tabular}{llll}
\hline Patient ID & Age (years) & Sex & BMI $\left(\mathbf{k g} / \mathbf{m}^{\mathbf{2}}\right)$ \\
\hline 1 & 63 & M & 29.8 \\
2 & 48 & M & 38.6 \\
3 & 43 & F & 29.4 \\
4 & 72 & F & 26.3 \\
5 & 33 & F & 23.4 \\
6 & 65 & M & 27.0 \\
7 & 58 & M & 30.0 \\
\hline
\end{tabular}

medications). Body mass index remained stable throughout. Patient demographics are in Table 1.

Medications were unchanged post-RDN, except for two patients. One patient, with no BP response post-RDN, had antihypertensive medications increased. Another patient was symptomatic with a marked BP improvement post-RDN, and his medication was reduced. The change in medication did not alter their post-RDN BP responder status.

Patients underwent RDN with the Symplicity catheter. Multiunit muscle sympathetic nerve activity (MSNA) was measured from the peroneal nerve. Sympathetic baroreflex sensitivity (sBRS) was measured at baseline, 1, and 6 months post-RDN. One patient did not undergo MSNA at 1 month.

The sBRS was calculated by threshold baroreflex analysis, associating spontaneous fluctuations in diastolic BP (increases and decreases) to the occurrence of spontaneous MSNA bursts. Sensitivity is defined as the slope of the linear regression analysis.

Results Response to RDN was defined as a fall in SBP $\geq 10 \mathrm{~mm} \mathrm{Hg}$. Four of the seven patients were responders at 1 and 6 months post-RDN. One patient was a BP non-responder at 1 month but responded by 6 months. Another patient was initially a BP responder, but a non-responder at 6 months.

The sBRS improved at $1(\Delta-1.7 \pm 0.5 \% / \mathrm{mm} \mathrm{Hg}, \mathrm{p}<0.05, \mathrm{n}=6 / 6)$ and 6 months $(\Delta-0.9 \pm 0.3 \% / \mathrm{mm} \mathrm{Hg}, \mathrm{p}<0.05, \mathrm{n}=6 / 7)$ post-RDN (figure $1 \mathrm{~A}$ ). Figure $1 \mathrm{~B}$ shows the change in the total sBRS for a single patient.

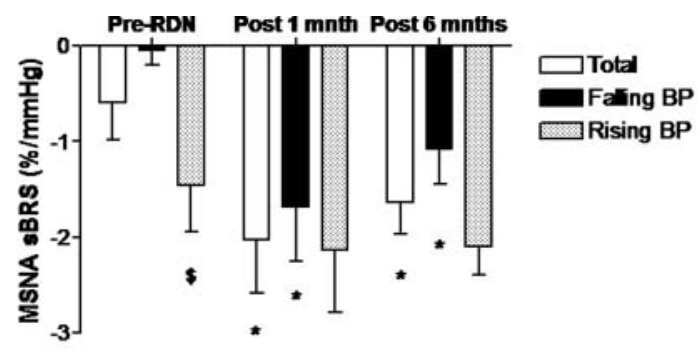

\section{RENAL DENERVATION IMPROVES AUTONOMIC RESPONSES TO FALLS IN BLOOD PRESSURE}

LE K Ratcliffe, ${ }^{1}$ E C Hart, ${ }^{1}$ A E Burchell, ${ }^{1}$ A Baumbach, ${ }^{2}$ J F R Paton, ${ }^{1}$ A K Nightingale ${ }^{2}{ }^{1}$ University of Bristol; ${ }^{2}$ Bristol Heart Institute

doi:10.1136/heartjnl-2013-304019.130

Introduction Renal denervation (RDN) decreases blood pressure (BP) in some patients with resistant hypertension. Little is known about the mechanisms through which the BP fall is achieved. It is thought that decreased afferent renal input to the brainstem results in a fall in central sympathetic outflow. We hypothesised that sympathetic baroreflex sensitivity (sBRS) would improve following RDN.

Methods We studied seven patients with resistant hypertension (clinic systolic $\mathrm{BP}>140 / 90 \mathrm{~mm} \mathrm{Hg}$, on $\geq 3$ anti-hypertensive 
Baroreflex function is dependent on whether $\mathrm{BP}$ is rising or falling, therefore we analysed the MSNA data for rising and falling BP separately (Figure 1A). Sympathetic BRS during falling BP was significantly improved following $\mathrm{RDN}$ in patients at both $1(\Delta$ $-1.5 \pm 0.7 \% / \mathrm{mmHg}, \mathrm{p}<0.05, \mathrm{n}=4 / 6)$ and 6 months $(\Delta-1.8 \pm 0.4 \% /$ $\mathrm{mm} \mathrm{Hg}, \mathrm{p}<0.05, \mathrm{n}=6 / 7)$. In contrast, the sympathetic BRS to rising BP was unchanged at both 1 and 6 months post-RDN.

Conclusions Four of the seven patients with resistant hypertension had a BP response to renal denervation. The sBRS to falling, but not rising, $\mathrm{BP}$ improved following $\mathrm{RDN}$. This is important since improved baroreflex control of falling BP might help to reduce orthostatic hypotension. This is particularly relevant in patients on multiple anti-hypertensive medications. The mechanisms underpinning the improved baroreflex are unclear, but may provide a relatively early marker of procedural success in those patients that respond to RDN. 